\title{
The future of scientific communication in pathology
}

\author{
John Crocker
}

The exchange of scientific information in a constructive, non-competitive way is one of the joys of being a pathologist, especially when involved intensively in the academic world. Whether such ideals are always achieved is debatable; however, the advent of "electronic communication" should impress upon us all that such exchange of information should be rapid, robust, and reliable in terms of content. The latter consideration leads directly to the currently widely promulgated field of "evidence based" medicine and pathology.

Communication between pathologists, from all specialties, is clearly essential. This is true both within and between the areas encompassed in the field. For example, I should find myself lost (as a lymphoma pathologist) if I did not have everyday contact with my colleagues who are haematologists. Such a relationship enables rapid and (I hope!) accurate clinicopathological decisions about patient management; furthermore, in such a context, we often arrive at ideas for research, much of which is conducted at a fundamental level. How much, then, of what we read or hear can we believe? This question inevitably leads to the fashionable topic of "evidence based pathology."

\section{"Evidence based pathology"}

What on Earth does this mean ${ }^{12}$ Surely all that we do or believe in pathology is based on good, solid evidence. This is certainly so in many if not most cases, but anecdotal comment and ideas abound. For example, I was warned as a trainee that fresh water should never be allowed to contact unfixed tissues, as it would destroy good histological morphology. However, this was shown not to be true. Another example: we were told that delayed fixation of tissues should lead to diminished mitotic scores, as cells complete their cycle in that time: again, not necessarily true! Is there really evidence for the proposed sequence of $B$ cell differentiation on which much of our current classification of lymphomas is based? The examples are endless and those quoted are from the "scientific" pole of medicine, far distanced from the more clinical end of the spectrum, where, one assumes, there is much more opinion than fact. (Note that I have no evidence for this comment!)

A facility which will certainly impinge on us all is the Cochrane Library. ${ }^{3}$ This is an enormous resource, available electronically, which is attempting to avoid biases in the analysis of research studies. For example, there is a widespread tendency for the presentation of positive rather than negative findings at meetings and in print. Furthermore, the former tend to be published in more prestig- ious, widely read journals than the latter. In addition, the Cochrane Library will register new research. Clearly, this is an exciting new project, with multiple databases of medical information, which is likely to expand very rapidly.

Minimum datasets are transforming our way of reporting cancer specimens in histopathology. One assumes (or at least, hopes) that the reasons for our accepting such guidelines are robust and relevant, as much time has been added to our busy lives in conforming to such protocols. Again, it is presumed that the proscribed protocols for our daily work practice are correct but on what evidence?

Not surprisingly, much of the study of evidence based pathology has been performed in haematology and clinical chemistry, where results are in the form of numerical data and, although these may vary on an inter- and intralaboratory level, they are measurable and objectively verifiable. However, the significance of such values is often open to interpretation. In histopathology, observations and their management are inevitably much more subjective. Fields that cause problems abound and include the grading of cervical intraepithelial neoplasia (CIN), the assessment of the Rye subtype of Hodgkin's disease, and so forth. It would have been hoped that the advent of immunocytochemistry might lead to objectivity in this field; unfortunately, however, this is sometimes far from the truth. We adhere to a range of grading and staging methods in our reports but are we sure that these are useful? For example, in CIN it may be that the type of HPV responsible for the disease is more important than the conventional grading system. Furthermore, in Hodgkin's disease, the presence or absence of Epstein-Barr virus may afford information related to response to treatment and yet the assessment of EBV status is rarely considered by the histopathologist. One major problem with the internet lies in the fact that information placed in it may have little or no basis in evidence (see below).

\section{The internet}

There is an established tradition of "on-line" information available to the scientific and medical community, in the form of such resources as MEDLINE, OVID, and so on, which facilitate the search for published references. Like any other such media, these rely on regular updating and are relatively limited in their content. However, with the advent of the world wide web or internet, the amount of information available to the subscriber has increased by numerous orders of magnitude. It seems likely that much of our knowledge will 
become available in the future through the web $^{4-6}$; however, just as paper books depend on being up to date, even more so do electronic publications. The advantage of the latter over the former is that on-line media can be updated so frequently and the result seen by the recipient. Accuracy of medical information on the net is being questioned but, as above, I suspect that it is at no more risk of inaccuracy of content than is the printed word. For example, some 10 years ago, I accidentally conducted an experiment in misinformation, which was, fortunately, trivial. In the early days of our investigation of interphase nucleolar organiser regions, as demonstrated by means of their argyrophilia, I stated in a paper that "NORs are loops of DNA .....". ${ }^{7}$ This is not quite true; NORs are segments of DNA. Despite this inaccuracy, I am forever refereeing manuscripts which quote the former statement. This underlines the fact that authors should always refer to the original papers, rather than quoting subsequent writing! This would, of course, apply as much to the internet as to paper media. Perhaps we are frightened of a new medium and of its vast content, which could readily pollute an already rather unreliable literature simply because of its enormous scale.

The internet offers many facilities to the pathologist. These include massive databases, such as those detailing CD designated molecules, histology archives, hepatitis viruses, genome structures (including the human genome project!), and a myriad further topics. Most can be downloaded for subsequent reference on to paper, which remains, in my opinion, a very convenient medium.

The structure of the internet has been reviewed in many publications and it is unnecessary to go into great detail here; nonetheless, a brief outline follows. Sites have a URL (universal resource locator) which allows access to their content. Such URLs are opened through various "search engines," which are of variable efficiency in locating the desired URL. Most of us now have access to a computer and therefore can enter the URL which we require, either at home or work.

Once into the net, what does it offer us? The answer is that there is very much out there. For example, there is undergraduate teaching material, which may be relatively expensive but still worthwhile. Also, histopathology atlas-type illustrations are available and, as above, there are databases of CD designated proteins, oncogenes, and so on. Another part of the internet is that now related to continuing professional development (CPD) and this is discussed below. One hopes that the ability rapidly to correct errors in information on the internet will overcome the problem of publishing, in that immediately a paper is in print it is senescent. Unfortunately, the presence of this medium has led to yet more Americanisation of English, together with the jargon associated with management training; however, perhaps correct and logical grammar will prevail.

\section{Publishing}

It is now quite common to buy either student or postgraduate textbooks which come with an attached CD-ROM relating to their contents. These are of variable quality and may either enhance the paper content or, in rare cases, insult the readers' intelligence! Again, this medium could find great usefulness in the future. For instance, we live in an age where consent for necropsy is not readily forthcoming. However, thanks to the Sheffield group, it is now possible to buy a "virtual postmortem." At the molecular level, simulations of real time PCRs are also at hand and there are even catalogues advertising numerous machines and methods. As an editor of a BMF journal, I am only too aware of the pressures on the field in relation to the input of papers and their turnround times. Thus we can envisage the acceptance of manuscripts over the internet, and the production of figures by means of electronic media is already accepted.

Currently, we are seeing the publication of books and journals with an adjunct in the form of internet links. These latter may be "enhanced" in relation to the paper version of the publication, in that video and sound may be added. Thus, for example, a book related to immunology might have animations of antigen/ antibody binding. Furthermore, texts regarding laboratory techniques can show them in video format. Therefore a dynamic aspect of otherwise static information can be attained. In addition, it is now potentially possible to evaluate laboratory equipment from a distance without the inconvenience of full on-site demonstration. I have a subscription to the journal Science and as part of this it is possible to view enhanced or expanded versions of some of the paper edition.

Another field related to communication is that of peer review of scientific and clinical papers. ${ }^{8}{ }^{9}$ This subject is currently under much scrutiny and a large amount of our assessment of the process is subjective and uncertain. I hope and suspect that "open" or nonanonymous refereeing of manuscripts will become widespread, if not universal. As with the submission of abstracts to academic meetings, the perennial problem of assessor bias should be overcome by anonymity of the authors. This would diminish any conflict of interest with regard to both authors and referees. Perhaps a more radical if not iconoclastic approach could be adopted, namely that of discarding the process of peer review entirely! This would generate a vast amount of information, either spurious or credible, and would be unwieldy on paper but might be possible electronically. It would certainly lead to a very lively and, on occasion, passionate correspondence and debate; perhaps we should all consider this option, especially as it would be facilitated and speeded by the net and not create vast expanses of paper!

Teleconferencing and telecommunication If one works in a large department, with colleagues who have a wide spread of specialist interests, then obtaining a good second opinion 
on a difficult clinical case causes no problem. However, on occasion, an isolated pathologist may need help, as may a group such as that above, if the specimen originates from a "superspecialised" area. At present, this can partly be expedited by means of not simply sending away the specimen geographically but by the use of one to one discussion in "real time" or after a short delay. The demands leading to such a facility are to no small extent driven by the prevailing clinical obsession with speed of reporting, which is manifestly of questionable wisdom.

Imaging can be of "static" type, where images are sent to the recipient and there is no possibility for response to those images. ${ }^{10}$ Of course, the sending of such visual information would depend on selectivity by the sender and would be limited by gross bias. Also, the recipient would be limited by lack of control over the material received. This underlines the advantages of interactive telepathology, where the recipient has control over the sighting of the images received. Thus, for example, in histopathology, the receiving pathologist could control and manipulate the fields of the slide of interest. However, the technology available to us at present has the constraint that this process is fairly slow. Like anything else here, this will be speeded. ${ }^{11}$

\section{The future}

In these somewhat paranoia inducing days of accountability, when management speak such as "governance" abounds, we shall surely be under increasing pressure to prove that we communicate. CPD will play an expanding role in our lives and consume more of our time. Interactive forms of CPD, such as those involving CD-ROM technology, especially of the writable type, will be widespread.

On a day to day basis, in the clinical environment I am sure that teleconferencing will come to play a major role in the referral of difficult histopathological specimens and that in, for example, clinical chemistry and haematology, reference values and data could be assessed by expert panels in this way. Electrophoretic gels could be scanned and sent to other experts for comment, and DNA "fingerprints" could be compared with reference databases, even in a forensic context.
I am enabled, by being asked to write this brief informal review, to express my growing concern about communication of pure research within our profession. Ideally, this should be open and freely exchanged; however, in the real world-where careers, finances, and status depend on publication numbers and even qualities-secrecy is widespread with regard to ideas and data. Perhaps a more insidious and worrying development is that of "intellectual property." This concept implies that should a researcher working for a hospital trust or university come up with a potentially commercially interesting technique or idea, then it becomes the property of the latter. This will surely tend to stifle open exchange of academic thoughts and collaboration.

Finally, where will all of this end? Perhaps in the year 3000 we will all have cerebral implants to exchange information! Perhaps in the year 3000 there will be no disease. Seriously, there is much to be said for face to face communication, commonly known as "talking." I enjoy this regularly at the Path Soc meeting in January and July each year. This journal is linked to the $\mathrm{ACP}$, and their meetings are, of course, important in another channel - a much more clinical context. The meetings of the ACP are arguably the last bastion of the Pathologist rather than The pathologist!

I am indebted to my wife, Dr The Hon Catherine Crocker, for pointing me in the direction of The Cochrane Library!

1 Booth A. Mapping the evidence base of pathology. $F$ Pathol 1999;188:344-50.

2 Fleming KA. Evidence-based pathology. F Pathol 1996;179: 127-8.

3 Chalmers I. Cranberry juice and the Cochrane Library. MRC News 1998;79:17-19.

4 Rashbass J. Why use the internet to teach pathology? f Clin Pathol 1998;51:179-82.

5 Weinstein S. Internet for pathologists. Broadsheet No 46. Pathology 1998;30:364-8.

6 Rashbass J. Molecular biology and the Internet. $\mathcal{F}$ Clin Pathol Mol Pathol 1996;49:136-9.

7 Crocker J, Nar P. Nucleolar organizer regions in lymphomas. F Pathol 1987;151:111-18.

8 Page G. Research communication in the 21 st century. Eur Sci Editing 1999;25:51-3.8.

9 Goodlee F, Jefferson T. Peer review in health sciences. London: BMJ Publishing Group, 1999.

10 Leong W-M, Graham AK, Gahn T, et al. Telepathology: clinical utility and methodology. In: Lowe DG, Underwood JCE, eds. Recent advances in histopathology. Edinburgh: Churchill-Livingstone, 1999:217-40.

11 Sowter C, Wells CA. Telepathology: assessment of the implications and applications of telepathology for practical diagnostic pathology. F Clin Pathol 1998;51:714-15. 